\title{
A systematic review and meta-analysis of gastrointestinal events associated with nonoperative therapies for neuroendocrine tumors
}

This article was published in the following Dove Press journal:

OncoTargets and Therapy

\section{Quhui Wu' $u^{1, *}$ \\ Bo Chen ${ }^{2, *}$ \\ Guofu Yan' \\ Zhulin Yang ${ }^{2}$ \\ Li Xiong ${ }^{2}$ \\ Jun $\mathrm{He}^{2}$}

'Graduate School, Hunan University of Chinese Medicine, Changsha 410208, China; ${ }^{2}$ Department of General Surgery, Second Xiangya Hospital, Central South University, Changsha 4I00II, China

*These authors contributed equally to this work
Correspondence: Li Xiong; Jun He Department of General Surgery, Second Xiangya Hospital, Central South University, I 39 Renming Rd, Changsha 4I00 II, China

Tel +86 I8। 073। 1987;

+86 I5084740650

Email lixionghn@csu.edu.cn; junjunhe@csu.edu.cn

\begin{abstract}
The risk of gastrointestinal (GI) events induced by nonoperative therapies in patients with neuroendocrine tumors (NETs) is unclear. Nonoperative therapies include somatostatin analogs, molecular targeted agents, cytotoxic chemotherapy, interferon- $\alpha$, and peptide receptor radionuclide therapy. We undertook an up-to-date meta-analysis to determine the incidence and relative risks (RRs) of GI events in NET patients treated with these therapies. MEDLINE, EMBASE, the Cochrane Central Register of Controlled Trials, and ClinicalTrials.gov were searched to identify relevant trials. Eligible trials were selected according to the PRISMA statement. Summary incidence, RR, and 95\% CIs were calculated using random- and fixedeffects models. We included 2,890 patients from 17 randomized controlled trials in this meta-analysis. The experimental treatments led to increased incidence and risks of GI events compared to the control treatments $(P<0.05)$. Diarrhea was the most common GI event. The experimental treatments were associated with increased risks of high-grade nausea (RR 2.36; 95\% CI 1.05-5.25; $P<0.01$ ) and vomiting (RR 1.89; 95\% CI 1.04-3.44; $P<0.05$ ). In regard to specific therapy regimens, everolimus led to increased risks of diarrhea (RR 2.97; 95\% CI $1.83-4.83 ; P<0.05$ ), vomiting (RR 2.19; 95\% CI 1.38-3.48; $P<0.05$ ), and anorexia (RR 3.20; 95\% CI 1.69-6.06; $P<0.05$ ), whereas VEGFR inhibitors led to increased risk of diarrhea (RR $2.12 ; 95 \%$ CI $1.39-3.25 ; P<0.05)$. Additionally, GI NETs led to higher risk of GI events than pancreatic NETs. Thus, nonoperative therapies are associated with increased risks of GI events in NET patients, and rigorous management is warranted to minimize the adverse impact on treatment outcomes and to improve quality of life
\end{abstract}

Keywords: chemotherapy, somatostatin analogs, everolimus, neuroendocrine tumors, metaanalysis, gastrointestinal events

\section{Introduction}

Neuroendocrine tumors (NETs) originate from cells across the diffuse endocrine system and include a variety of subtypes, such as pancreatic, gastrointestinal (GI), lung, and thymus NET. ${ }^{1}$ The incidence of NETs in the US was 6.98 cases per 100,000 people in $2012 .{ }^{2}$ Treatment guidelines for NETs recommend that therapeutic options for advanced tumors and distant metastasis involve somatostatin analogs (SSAs), molecular targeted agents (eg, everolimus), cytotoxic chemotherapy, interferon- $\alpha$ (IFN- $\alpha$ ), and peptide receptor radionuclide therapy (PRRT), as well as surgery in some cases involving limited metastases. ${ }^{1}$ This study excluded surgery, and only the abovementioned therapies (designated as "nonoperative therapies") are included in the meta-analysis. 
Hormonal hypersecretion that is derived from NETs may or may not lead to corresponding symptoms. For example, one of the most common symptoms of carcinoid syndrome (which can occur in NET patients) is diarrhea. ${ }^{3}$ However, diarrhea is a frequently reported side effect of sunitinib and everolimus. ${ }^{4,5}$ In addition, other GI events, including nausea, vomiting, and anorexia, are also associated with NET treated with cytotoxic chemotherapies, SSAs, and PRRT. ${ }^{6-8}$ In these scenarios, when NET patients are treated with those therapies, the GI events might be exacerbated by the very common effects of the NETs themselves, especially for patients with characteristics of gastroentero-active hormone hypersecretion. Thus, optimizing therapeutic options by reducing GI events is necessary for these patients. Furthermore, early recognition and appropriate management of GI events are helpful to avoid inessential dosage reductions and temporary or permanent treatment discontinuations among NET patients. In this study, we sought to thoroughly investigate the incidence and the relative risks (RRs) of GI events in NET patients receiving nonoperative therapies, including SSAs, molecular targeted agents, cytotoxic chemotherapy, IFN- $\alpha$, and PRRT, via a meta-analysis of available randomized controlled trials (RCTs).

\section{Acquisition of relevant studies}

This study adhered to the PRISMA guidelines. ${ }^{9}$ To select relevant studies, we retrieved trials from MEDLINE, EMBASE, and the Cochrane Central Register of Controlled Trials (CENTRAL). In addition, we searched ClinicalTrials. gov to further detect relevant trials. The preliminary retrieval of relevant trials was conducted on July 9, 2018 by a single reviewer (WQ).

The search strategy for MEDLINE was as follows. With regard to the selection of relevant patients, the following $\mathrm{MeSH}$ and non-MeSH terms were used: "neuroendocrine tumor" or "tumor neuroendocrine" or "tumors neuroendocrine" or "insulinomas" or "islet cell carcinoma" or "gastrinomas" AND "pancreatic" or "pancreas" or "stomach" or "gastric" or "intestinal" or "intestine" or "digestive track" or "gastrointestinal tract" or "GI tract" or "gastroenteropancreatic". Regarding selecting relevant interventions, the following MeSH and non-MeSH terms were used: "therapy, drug" or "drug therapies" or "therapies drug" or "chemotherapy" or "chemotherapies" or "pharmacotherapy" or "pharmacotherapies" or "streptozotocin" or "fluorouracil" or "doxorubicin" or "dacarbazine" or "temozolamide" or "interferon" or "everolimus" or "somatostatin analogs" or "octreotide" or "etoposide" or "streptozocin" or "dacarbazine" or "cisplatin" or " 177 Lu-DOTATATE" or "peptide receptor radionuclide therapy" or "PRRT". With regard to selecting the relevant types of studies, the following terms were used to obtain RCTs: "randomized controlled trial.pt" or "controlled clinical trial.pt" or "randomized.ab" or "placebo.ab" or "clinical trials as topic.sh" or "randomly.ab" or "trial.ti". Ongoing trials were searched with the terms "neuroendocrine tumor" AND other terms including "chemotherapy" or "somatostatin analogs" or "interferon" or "everolimus" or "sunitinib" or other potential efficient therapies. We only included trials involving humans. When an English version of an article was unavailable, the article would be excluded.

\section{Eligibility criteria}

All of the potential publications were independently screened by two reviewers (QW and BC). Duplicates were excluded. Reviews, meta-analyses, conference abstracts, letters, editorials, and protocols were also excluded. Any publications that did not concern nonoperative therapies, NET, and GI events were eliminated. We removed studies involving nonrandomized designs, phase I trials, retrospective studies, subgroup reports, and pilot studies. Only studies that met the following criteria were further taken into consideration: 1) RCTs including phase II/III trials of NET patients with double-blind or open-label design; 2) randomized assignment of NET patients into Regimen A arm and placebo arm, or Regimen A+B arm and Regimen B arm; and 3) available data concerning GI events in both arms. The exclusion criteria were as follows: 1) studies involving $<20$ NET patients and 2) combined data on GI events, with no data on individual types of GI events. When multiple publications concerning the same trial were obtained, we prioritized recently published papers that presented the most data as relevant to our objectives. Full texts of eligible articles were obtained for the final determination of whether to include them, and any disagreements were discussed by the two reviewers until a consensus was reached.

\section{Data extraction and assessment of risk of bias}

Two reviewers (QW and JH) independently reviewed the full-text manuscripts of eligible trials and extracted the data using standardized Excel forms. Data on the name of first author, year of publication, study design, randomization method, basic patient characteristics, tumor location, tumor grading, experimental arm, control arm, treatment dosages and durations, and types and numbers of GI events (all grades 
and high grades [grades 3 or 4]) in each arm were extracted. We retrieved data on GI events (including diarrhea, nausea, vomiting, and anorexia) in each trial for this meta-analysis. Any discrepancy was resolved by discussion.

We assessed the quality of the included trials according to the Cochrane Collaboration checklist,$^{10}$ including: 1) method of randomization; 2) method of allocation concealment; 3) blinding of participants, clinicians, and data analysts; 4) presence of incomplete outcome data; and 5) selective outcome reporting.

\section{Statistical analysis}

Statistical analyses were conducted using Review Manager version 5.3 (Cochrane Collaboration) and Stata version 15. The principal summary measures were the incidence and RRs of GI events, with corresponding 95\% CIs, in each trial. To calculate the incidence of GI events, the number of each event (diarrhea, nausea, vomiting, and anorexia) and the number of patients in each arm were extracted from the safety profiles, while disregarding unrelated tumor types. The RRs and 95\% CIs of GI events for each trial were calculated by comparing the incidence of each GI event in the Regimen $\mathrm{A}$ and placebo arms or the regimens $\mathrm{A}+\mathrm{B}$ and $\mathrm{B}$ arms. Moreover, we calculated the RRs and corresponding 95\% CIs of high-grade (grade 3 or 4) GI events when the data were available.

The heterogeneity of the trials with regard to each variable used in the meta-analyses was tested using Cochran's $Q$ statistic, ${ }^{11}$ and heterogeneity was established on the basis of a $P$-value $<0.1$. We also used the $I^{2}$ statistic $[100 \% \times$ $(Q-d f) / Q$ ] to indicate whether there was heterogeneity between the studies by quantifying the percentage of the variability in effect estimates that was due to heterogeneity rather than chance. ${ }^{12}$ Heterogeneity was considered substantial when $I^{2}$ was $\geq 50 \%$. When $I^{2}$ was $<50 \%$ and $\geq 50 \%$, we conducted a meta-analysis using a fixed- and random-effects model, respectively.

We conducted prespecified subgroup analyses for each GI event, stratified by tumor type (pancreatic NETs, GI NETs, and NETs at other sites) and therapy regimens. Therapy regimens were stratified into mTOR inhibitors (everolimus and BEZ235), SSAs (pasireotide, lanreotide, and octreotide), VEGFR inhibitors (bevacizumab and sunitinib), IFN, cytotoxic chemotherapy (cisplatin, 5-fluorouracil, and capecitabine), and PRRT. A sensitivity analysis was conducted to check the robustness of the primary results by excluding low-quality trials (which involved excluding studies one at a time). We evaluated publication bias using funnel plots,
Egger's linear regression approach, and Begg's rank correlation test. ${ }^{13,14} \mathrm{~A}$ two-tailed $P$-value $<0.05$ indicated statistical significance.

\section{Results Search results}

Our search of the literature found 2,791 potentially relevant publications: 1,470 in MEDLINE, 1,239 in EMBASE, and 82 in CENTRAL. Figure 1 presents the processes and reasons for study selection. Among the initial publications, 642 duplicates were excluded. After reading the title, abstract, and full text, 17 RCTs, comprising nine phase III trials and eight phase II trials, were included in the metaanalysis.

\section{Study characteristics}

The basic characteristics of the included studies are listed in Table 1. This meta-analysis included a total of 2,890 patients (1,499 in the experimental arms; 1,391 in the control arms) from 17 RCTs. There were 1,303 patients with pancreatic NETs, 608 with GI NETs, 824 with gastroenteropancreatic NETs, 90 with lung NETs, and 65 with NETs at unknown sites.

Two trials evaluated the efficiency and safety of everolimus compared to placebo in pancreatic and GI NETs. ${ }^{15,16}$ One trial compared everolimus plus octreotide long-acting repeatable (LAR) with everolimus in NETs. ${ }^{5}$ One trial evaluated another mTOR inhibitor (BEZ235) in pancreatic NETs. ${ }^{17}$ Four trials assessed SSAs (pasireotide, octreotide, and lanreotide) in divergent conditions, either compared to placebo, compared to different SSA doses, or in combination with another regimen (in the experimental arm) as compared to the other regimen (in the control arm). ${ }^{18-21}$ Four trials assessed VEGFR inhibitors (sunitinib and bevacizumab) compared to placebo or in combination with everolimus to everolimus alone. ${ }^{4,22-24}$ Two trials evaluated IFN- $\alpha$ in NETs. ${ }^{7,25}$ Two trials assessed the safety of cytotoxic drugs (5-fluorouracil and cisplatin) in NETs. ${ }^{6,2}$ One trial evaluated the efficiency and safety of PRRT in midgut NETs. ${ }^{8}$

With regard to the trial therapy regimens, the everolimus dose was $10 \mathrm{mg}$ per day; BEZ235 dose was 400 or $300 \mathrm{mg}$ twice daily; pasireotide dose was $60 \mathrm{mg}$ per 28 days; octreotide dose was $30 \mathrm{mg}$ per month; lanreotide dose was $120 \mathrm{mg}$ per 28 days; sunitinib dose was $37.5 \mathrm{mg}$ per day; bevacizumab dose was $10 \mathrm{mg} / \mathrm{kg}$; IFN dose was $5 \times 10^{6} \mathrm{IU}$ three times per week; and ${ }^{177} \mathrm{Lu}$-Dotatate treatment involved $7.4 \mathrm{GBq}$. 


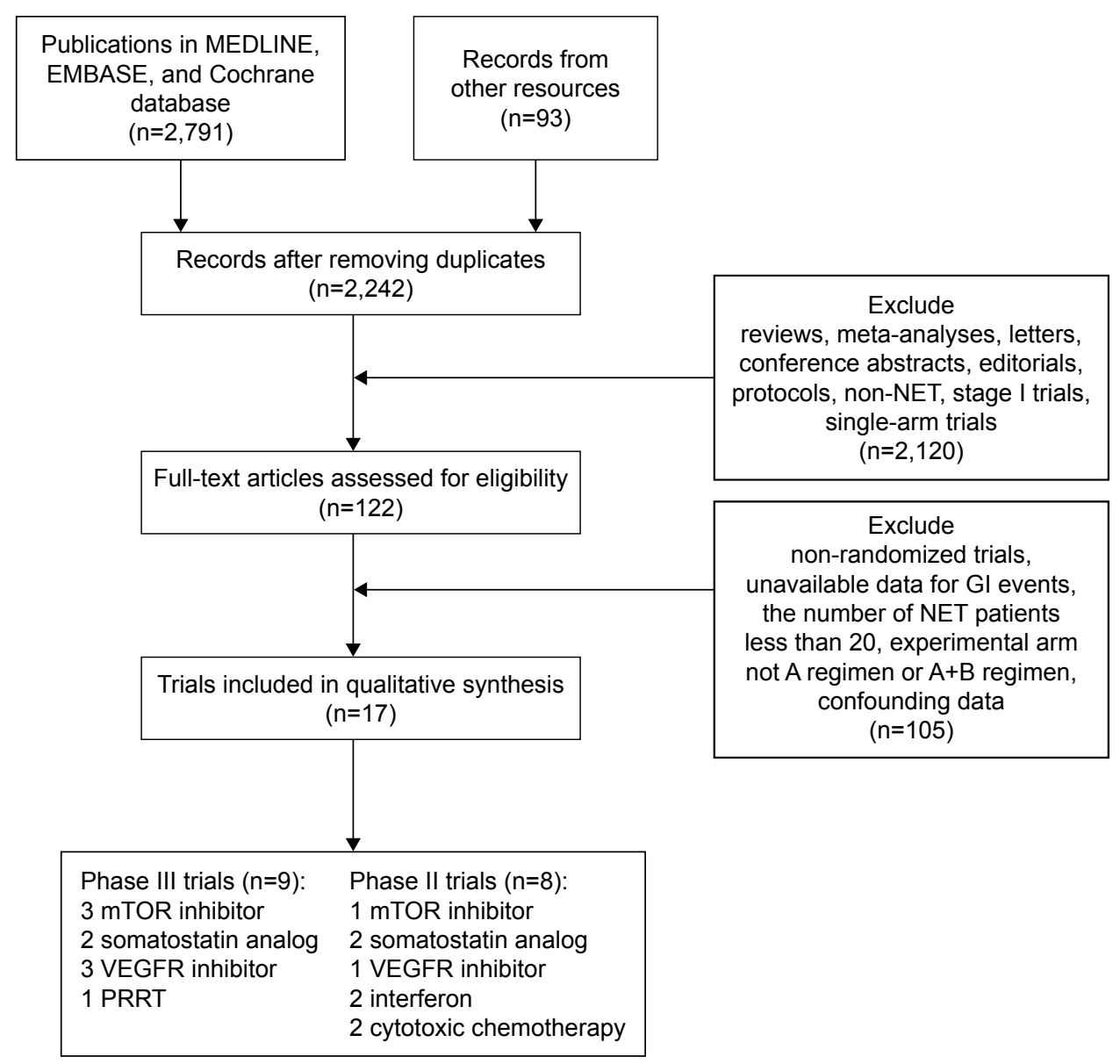

Figure I Processes and reasons for study selection.

Abbreviations: GI, gastrointestinal; NET, neuroendocrine tumor; PRRT, peptide receptor radionuclide therapy.

The sample sizes ranged from 31 to 429 patients, and five trials included $>200$ patients. In all trials, patients were randomly assigned to either the experimental or control arm. Ten trials reported the follow-up duration. All trials involved both male and female patients, with a mean age of $>53$ years.

\section{Risk of bias assessment}

All the included trials had randomized designs, with 14 trials providing details of the method of random sequence generation. With regard to blinding, eight trials were double-blind (in terms of participants and clinicians or treatment administration) and six trials had open-label designs (with no masking in either arm). Most of the trials reported all of the prespecified outcomes except two ongoing trials that lacked enough data to determine long-term efficiency and safety. Figure 2 illustrates the risk of bias assessments for all of the included trials. According to our assessments, the risk of bias summary suggested a moderate and acceptable bias in most domains, except for allocation concealment (which can lead to selection bias) and blinding of participants and personnel (which can lead to performance bias). Therefore, the overall quality of the included trials was generally acceptable.

\section{Incidence of $\mathrm{Gl}$ events}

Table 2 presents the incidence of GI events in the included trials. Incidence of any- and high-grade diarrhea in the experimental groups ranged from $1.2 \%$ to $62.0 \%$ and $0 \%$ to $7.3 \%$, respectively. The top three therapy regimens eliciting diarrhea in NET patients were everolimus plus pasireotide LAR, sunitinib, and BEZ235. The incidence of any-grade and high-grade nausea in the experimental groups ranged from $0 \%$ to $63.6 \%$ and $0 \%$ to $18.2 \%$, respectively. An incidence of nausea $>50 \%$ involved cisplatin plus capecitabine plus streptozocin, octreotide LAR plus ${ }^{177} \mathrm{Lu}$-Dotatate, and BEZ235. With regard to any-grade vomiting, the incidence ranged from $6.9 \%$ to $64.6 \%$ in the experimental groups. Data also suggested that cisplatin plus capecitabine plus streptozocin, octreotide LAR plus ${ }^{177} \mathrm{Lu}$-Dotatate, and BEZ235 were the treatments most likely to induce vomiting in NET patients. The incidence of any-grade anorexia ranged from $0 \%$ to $22.0 \%$ in the experimental groups. 


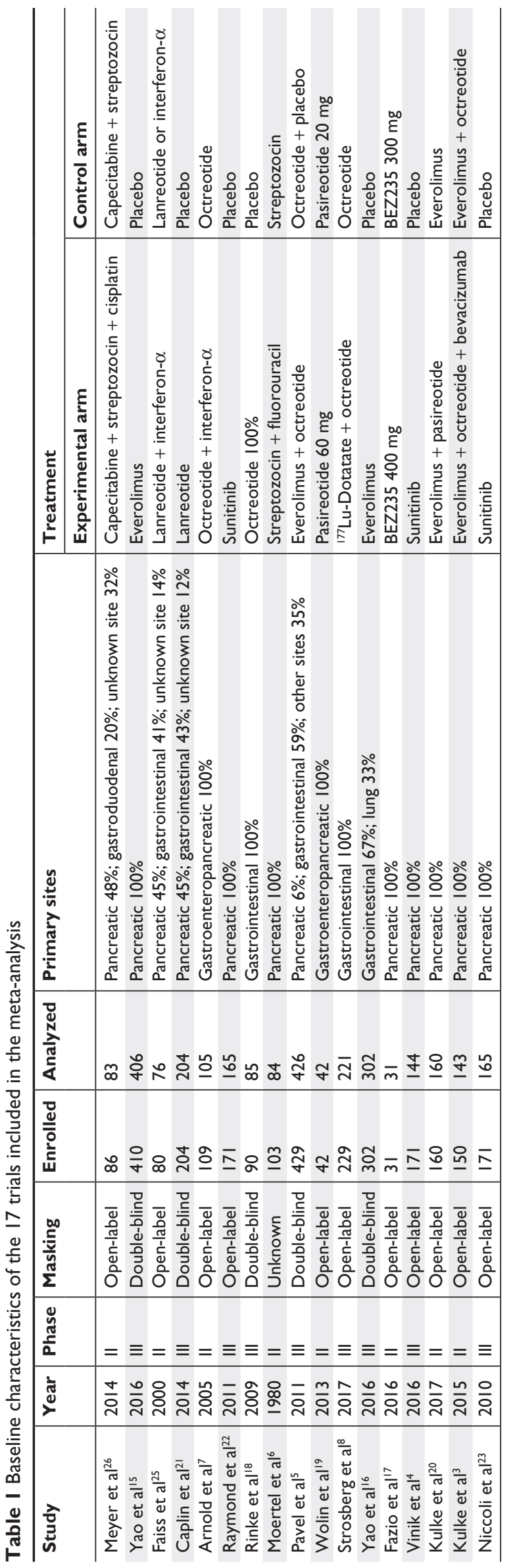

When considering the incidence of GI events stratified by therapy regimens, mTOR inhibitors (everolimus and BEZ235) led to any-grade diarrhea ranging from $33.8 \%$ to $54.5 \%$; any-grade nausea ranging from $17.1 \%$ to $54.5 \%$; any-grade vomiting ranging from $14.8 \%$ to $36.4 \%$; and any-grade anorexia ranging from $15.6 \%$ to $18.2 \%$. As for SSAs (pasireotide, octreotide, and lanreotide), the incidence of any-grade GI events were as follows: diarrhea ranging from $14.3 \%$ to $25.7 \%$; nausea ranging from $6.9 \%$ to $12.5 \%$; vomiting ranging from $0 \%$ to $6.9 \%$; and anorexia ranging from $0 \%$ to $6.3 \%$. Moreover, VEGFR inhibitors (sunitinib and bevacizumab) increased the incidence of any-grade diarrhea, nausea, vomiting, and anorexia, ranging from $1.2 \%$ to $60.0 \%, 2.4 \%$ to $45.1 \%, 2.7 \%$ to $34.1 \%$, and $4.1 \%$ to $22.0 \%$, respectively. In addition, the incidence of GI events for cytotoxic chemotherapy (cisplatin and 5-fluorouracil) was $43.2 \%$ for any-grade diarrhea, $63.6 \%$ for any-grade nausea, and $64.6 \%$ for any-grade vomiting.

\section{Overall RR of $\mathrm{Gl}$ events}

Sixteen RCTs were available to calculate the RRs of diarrhea; 446 diarrhea events were reported in 1,415 patients enrolled in the experimental groups, compared to 265 diarrhea events in 1,307 patients in the control groups. The pooled RR of any-grade diarrhea for nonoperative therapies vs controls was 1.55 (95\% CI 1.21-1.98, $P=0.0005)$, suggesting a significant increase in the risk of diarrhea for nonoperative treatments compared with controls. Similarly, the pooled RRs (encompassing eight trials) of any-grade nausea for nonoperative therapies vs controls was 1.55 ( $95 \%$ CI 1.14-2.10, $P=0.005$ ), suggesting a significant increase in the risk of nausea for nonoperative treatments compared with controls. The pooled RR (encompassing eight trials) of any-grade vomiting was 1.79 (95\% CI 1.08-2.97, $P=0.02)$ and the pooled RR (encompassing eight trials) of any-grade anorexia was 1.87 (95\% CI 1.22-2.87, $P=0.004$ ), suggesting that nonoperative therapies elicit significantly increased risks of vomiting and anorexia compared with controls. These results are presented in Figure 3.

The risks of high-grade GI events, including diarrhea and anorexia, associated with nonoperative therapies were not significantly increased when compared with the controls $(P>0.05)$. In contrast, the risks of high-grade nausea and vomiting were significantly increased $(P<0.05)$. The pooled RRs of high-grade GI events for nonoperative therapies vs controls were 1.69 (95\% CI 0.80-3.55) for diarrhea, 2.35 (95\% CI 1.05-5.27) for nausea, 1.89 (95\% CI 1.04-3.44) for vomiting, and 1.79 (95\% CI 0.26-12.05) for anorexia (Figure 4). Although the data indicated nonsignificant 


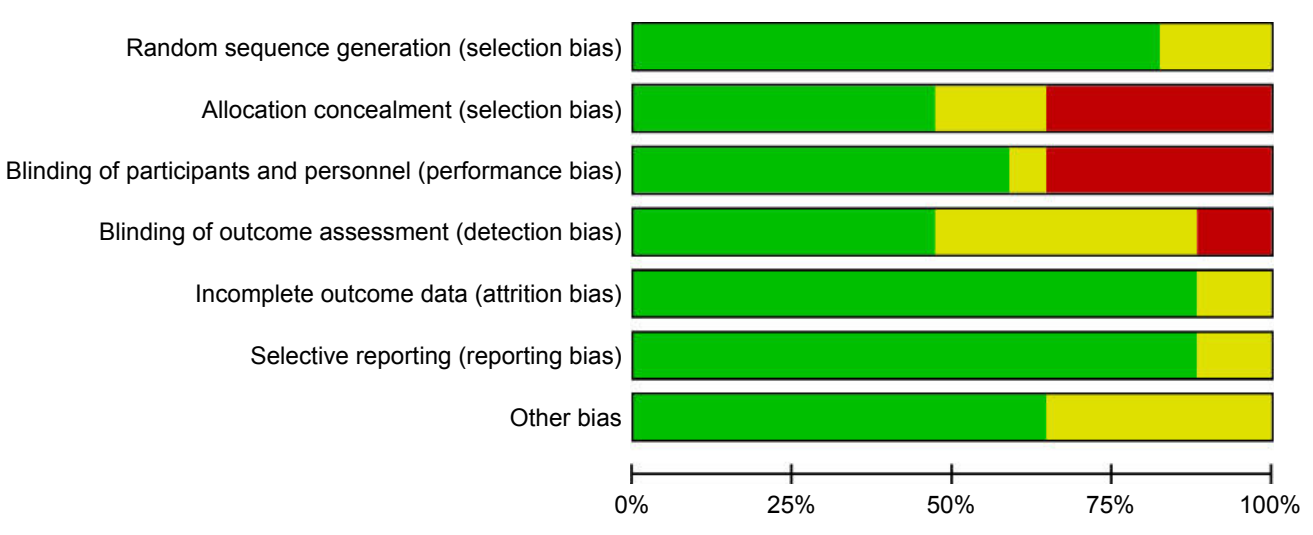

$\square$ Low risk of bias $\quad \square$ Unclear risk of bias $\square$ High risk of bias

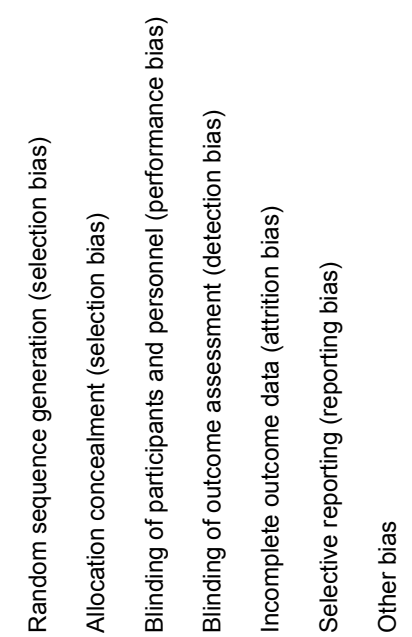

\begin{tabular}{|c|c|c|c|c|c|c|c|}
\hline Vinik et $\mathrm{al},{ }^{4} 2016$ & + & + & + & $?$ & $?$ & + & $?$ \\
\hline Rinke et al, ${ }^{18} 2009$ & + & $\odot$ & + & + & + & + & + \\
\hline Moertel et al, ${ }^{6} 1980$ & $?$ & $?$ & + & $?$ & + & + & $?$ \\
\hline Wolin et al,,$^{19} 2013$ & + & + & + & + & $\odot$ & + & + \\
\hline aymond et al,,$^{22} 2011$ & + & + & + & $?$ & + & + & + \\
\hline Yao et $a l,,^{15} 2016$ & + & + & + & + & + & + & $?$ \\
\hline Yao et $\mathrm{al}^{16} 2016$ & + & + & + & + & + & + & + \\
\hline Kulke et al, ${ }^{20} 2017$ & + & - & - & + & + & + & + \\
\hline Pavel et al, ${ }^{5} 2011$ & + & + & + & $?$ & + & + & + \\
\hline Caplin et al, ${ }^{21} 2014$ & + & + & + & + & + & + & + \\
\hline Kulke et al, ${ }^{3} 2015$ & $?$ & - & ก & - & + & + & + \\
\hline ,23 2010 & $?$ & $?$ & + & + & + & $?$ & $?$ \\
\hline Fazio et al, ${ }^{17} 2016$ & + & - & ? & ? & $?$ & $?$ & $?$ \\
\hline Arnold et al, 2005 & + & - & & $?$ & + & + & + \\
\hline Faiss et al, ${ }^{25} 2003$ & + & - & $(-)$ & & + & + & + \\
\hline Strosberg et al, ${ }^{8} 2017$ & + & - & - & + & + & + & + \\
\hline Meyer et al, ${ }^{26} 2014$ & + & $?$ & ? & $?$ & + & + & $?$ \\
\hline
\end{tabular}

Figure 2 Risk of bias assessments for all included trials. 


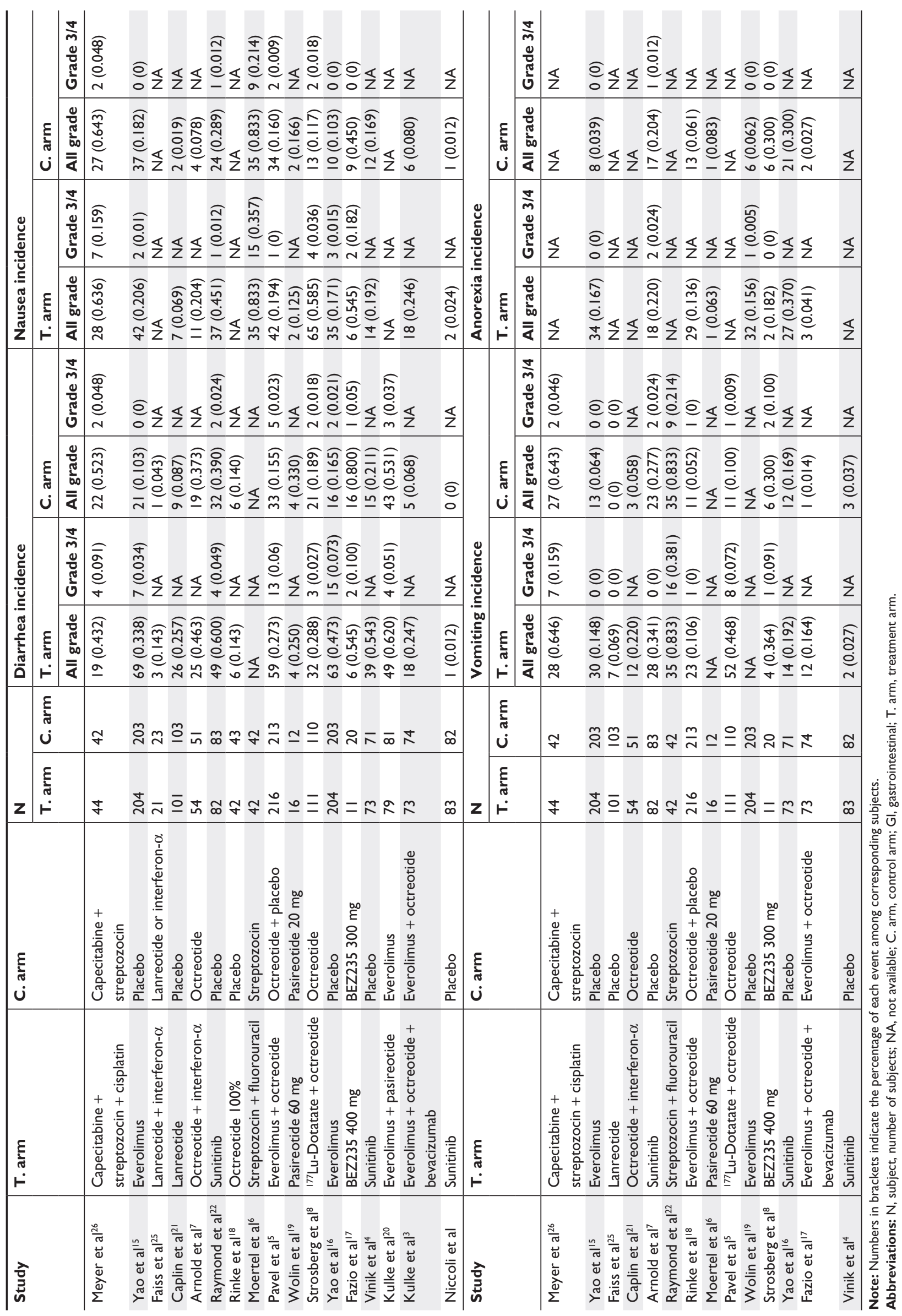


RRs $>1$ for diarrhea and anorexia, there were wide 95\% CIs (crossing 1).

\section{RRs of $\mathrm{Gl}$ events stratified by therapy regimen}

We conducted a subgroup risk analysis stratified by therapy regimen (Table 3 ). A total of 435 GI events occurred in 625 patients in the everolimus groups, compared to $239 \mathrm{GI}$ events in 513 patients in the control groups. The RR of any-grade GI events associated with everolimus was 1.47 (95\% CI 1.22-1.78, $P<0.0001)$. Although there was high heterogeneity in this meta-analysis $\left(I^{2}=69 \%\right)$, we omitted one study at a time to test the influence of each trial on the overall heterogeneity. When we omitted the trial by Yao et al, ${ }^{15}$ the heterogeneity of the included trials decreased to 0 . Thus, the risk of GI events due to everolimus compared with controls in NET patients significantly increased. SSA treatment in NETs did not lead to an increased risk of anygrade GI events $(P=0.28)$. Additionally, no significant difference in GI event risk was noted between the IFN- $\alpha$ and control groups $(P=0.24)$. The RR of any-grade GI events associated with combined chemotherapies vs single-drug

A

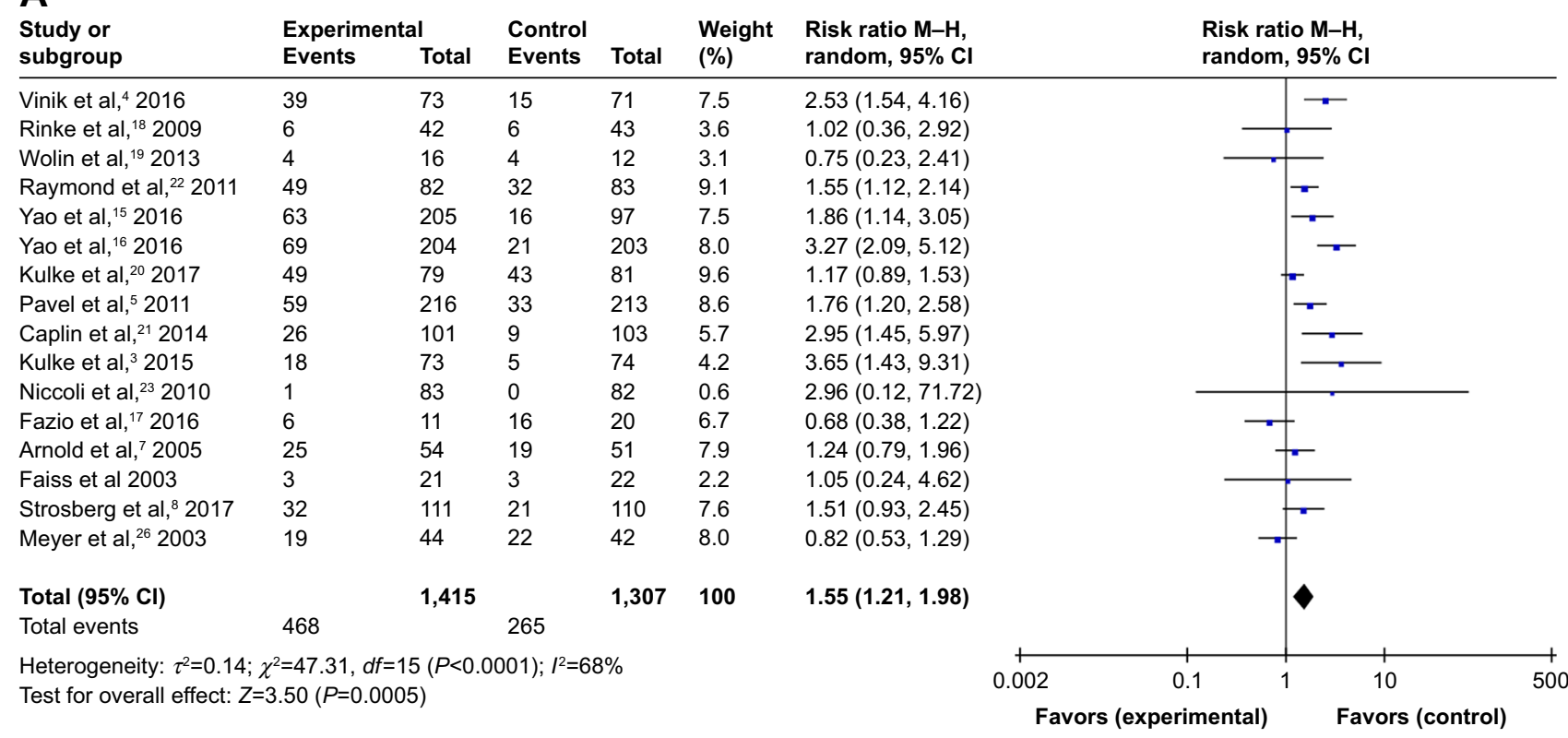

B

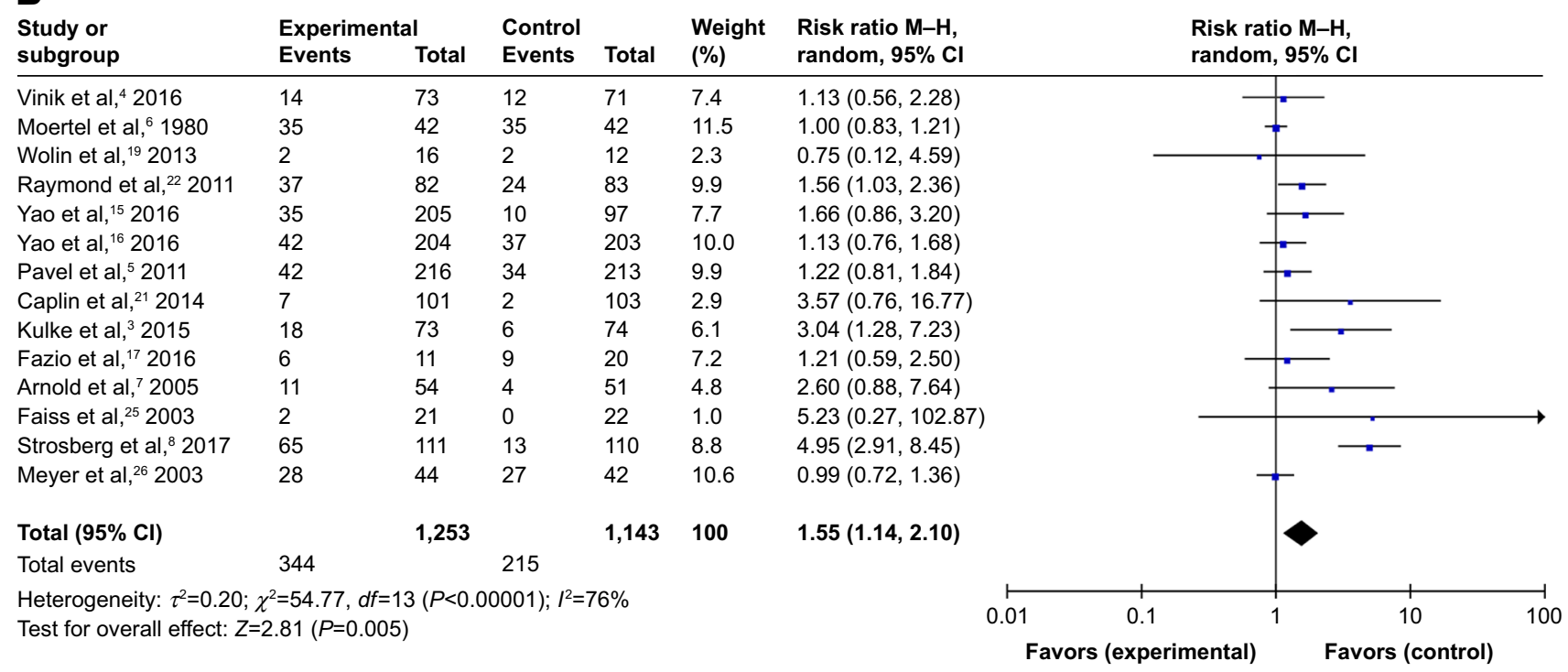

Figure 3 (Continued) 


\begin{tabular}{|c|c|c|c|c|c|}
\hline $\begin{array}{l}\text { Study or } \\
\text { subgroup }\end{array}$ & $\begin{array}{l}\text { Experimenta } \\
\text { Events }\end{array}$ & Total & $\begin{array}{l}\text { Control } \\
\text { Events }\end{array}$ & Total & $\begin{array}{l}\text { Weight } \\
(\%)\end{array}$ \\
\hline Vinik et al, ${ }^{4} 2016$ & 14 & 73 & 12 & 71 & 12.8 \\
\hline Moertel et al, ${ }^{6} 1980$ & 35 & 42 & 35 & 42 & 16.5 \\
\hline Raymond et al, ${ }^{22} 2011$ & 28 & 82 & 23 & 83 & 14.9 \\
\hline Pavel et al, ${ }^{5} 2011$ & 23 & 216 & 11 & 213 & 12.9 \\
\hline Kulke et al, ${ }^{3} 2015$ & 12 & 73 & 1 & 74 & 4.6 \\
\hline Arnold et al, ${ }^{7} 2005$ & 12 & 54 & 3 & 51 & 8.6 \\
\hline Strosberg et al, ${ }^{8} 2017$ & 52 & 111 & 11 & 110 & 13.7 \\
\hline Meyer et $a l,{ }^{26} 2003$ & 28 & 44 & 27 & 42 & 15.9 \\
\hline Total $(95 \% \mathrm{Cl})$ & & 695 & & 686 & 100 \\
\hline Total events & 204 & & 123 & & \\
\hline
\end{tabular}

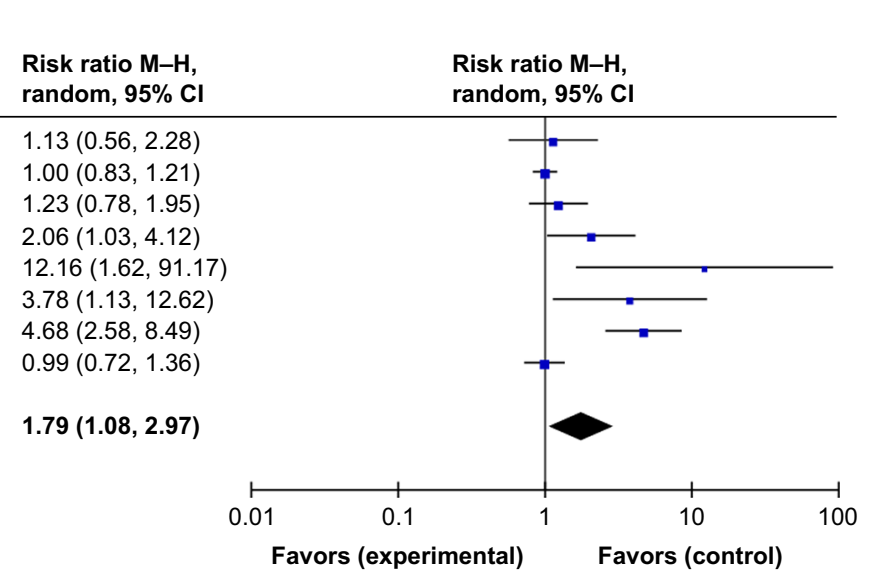

D

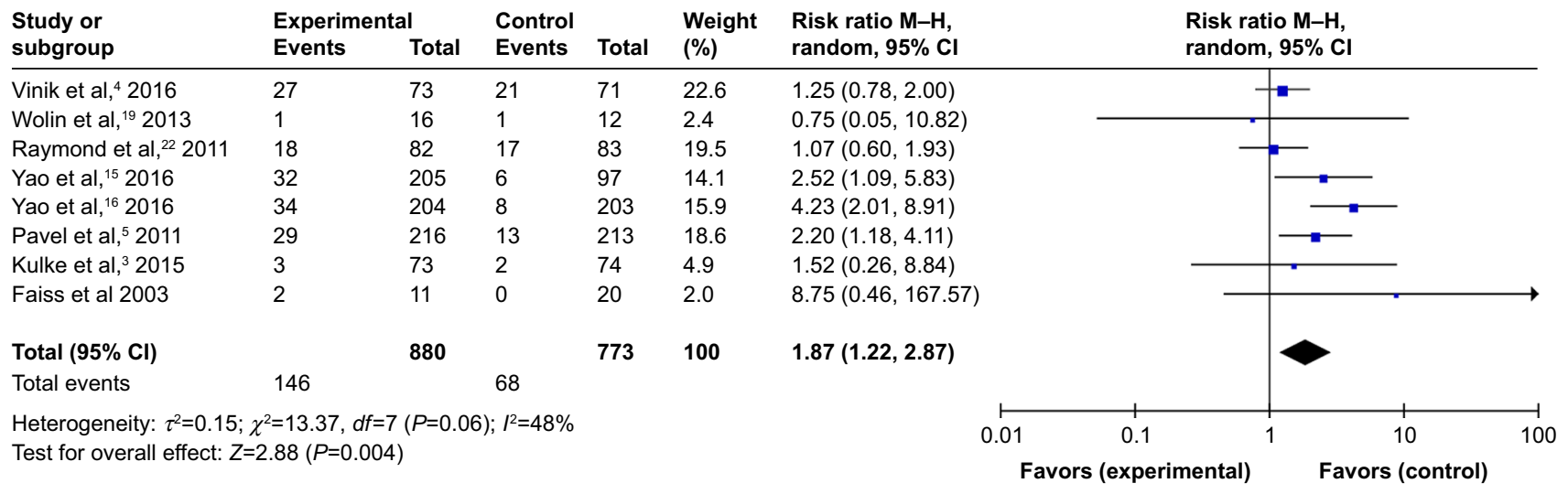

Figure 3 Relative risks of any-grade Gl events associated with nonoperative therapies.

Note: Relative risk associated with nonoperative therapies of any-grade (A) diarrhea, (B) nausea, (C) vomiting, and (D) anorexia.

Abbreviation: $\mathrm{Gl}$, gastrointestinal.

chemotherapy was not significant $(P=0.97)$, whereas the risk of high-grade GI events significantly increased (RR 1.97, 95\% CI 1.24-3.11, $P=0.004)$.

\section{RRs of Gl events stratified by tumor site}

We further conducted a meta-analysis of RRs of GI events stratified by tumor site (Table 4). In pancreatic NET patients, the RR was 1.77 (95\% CI 1.13-2.79, $P=0.01)$ for any-grade diarrhea, 1.39 (95\% CI $0.92-2.10, P=0.12)$ for any-grade nausea, 1.37 (95\% CI $0.88-2.13, P=0.17)$ for any-grade vomiting, and 1.48 (95\% CI 0.81-2.73, $P=0.21$ ) for anygrade anorexia. These pooled results indicated that nonoperative therapies significantly increased the risk of diarrhea in patients with pancreatic NET.

In GI NET patients, the RR was $1.66(95 \% \mathrm{CI}$ $1.05-2.63, P=0.03)$ for any-grade diarrhea, $1.57(95 \%$ CI $0.84-2.94, P=0.15)$ for any-grade nausea, $4.68(95 \% \mathrm{CI}$ $2.58-8.49, P<0.001)$ for any-grade vomiting, and 2.52 (95\% CI $1.09-5.83, P=0.03)$ for any-grade anorexia. Thus, the risks of GI events, except for nausea, significantly increased with nonoperative therapies in GI NET patients.
Several trials concurrently included pancreatic and GI NET patients, and the safety profiles were not reported by tumor site. The pooled RRs of these trials for diarrhea, nausea, vomiting, and anorexia were 1.56 (95\% CI $0.89-2.75$, $P=1.12$ ), 1.28 (95\% CI 0.88-1.87, $P=0.20), 2.16$ (95\% CI $0.84-5.60, P=0.11$ ), and 2.08 (95\% CI 1.13-3.83, $P=0.02$ ), respectively. Thus, the data from these trials only suggested an increased risk of anorexia due to nonoperative therapies in patients with gastroenteropancreatic NET.

\section{Publication bias}

The funnel plots did not show significant publication bias for any GI events. The $P$-values of Begg's test for the association between nonoperative therapies and risks of diarrhea, nausea, vomiting, and anorexia were 0.507, 0.876, 0.745, and 0.445 , respectively (Figure S1).

\section{Discussion}

Studies on NETs mostly concern the efficacy and safety of certain therapies (eg, octreotide LAR) and surgical management, as well as the verification of the use of Ki-67 


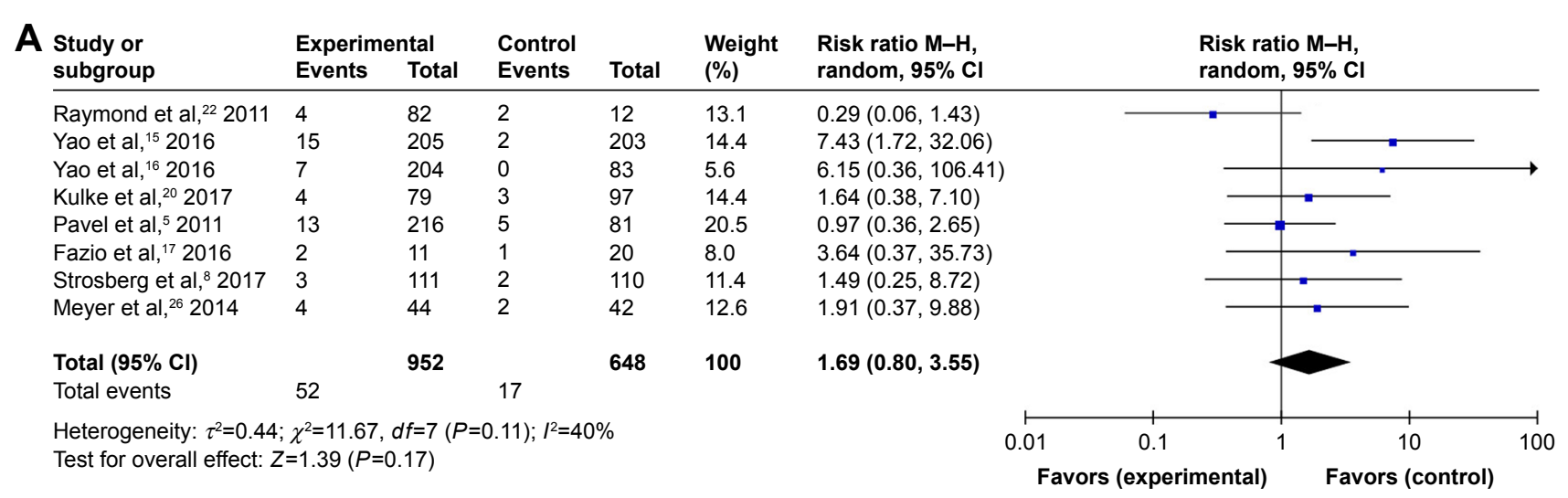

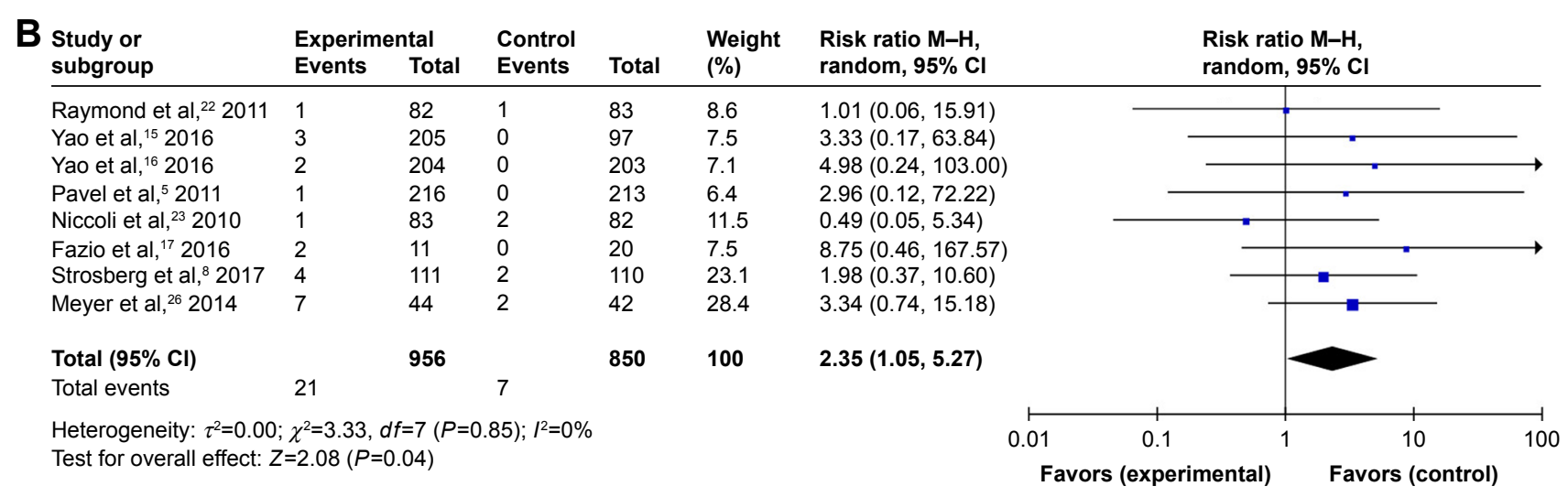

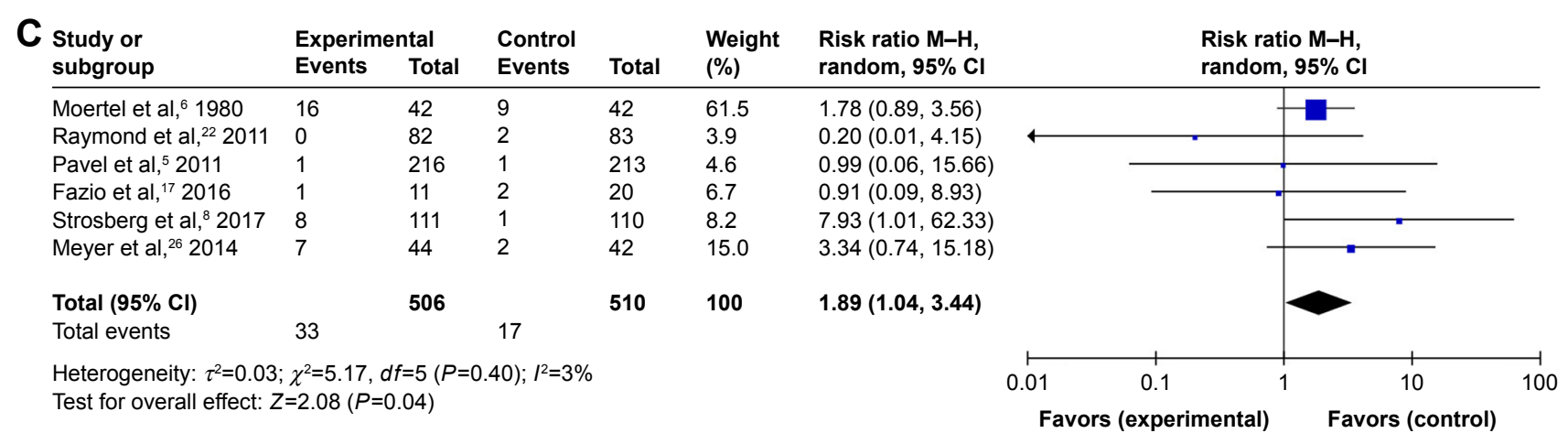

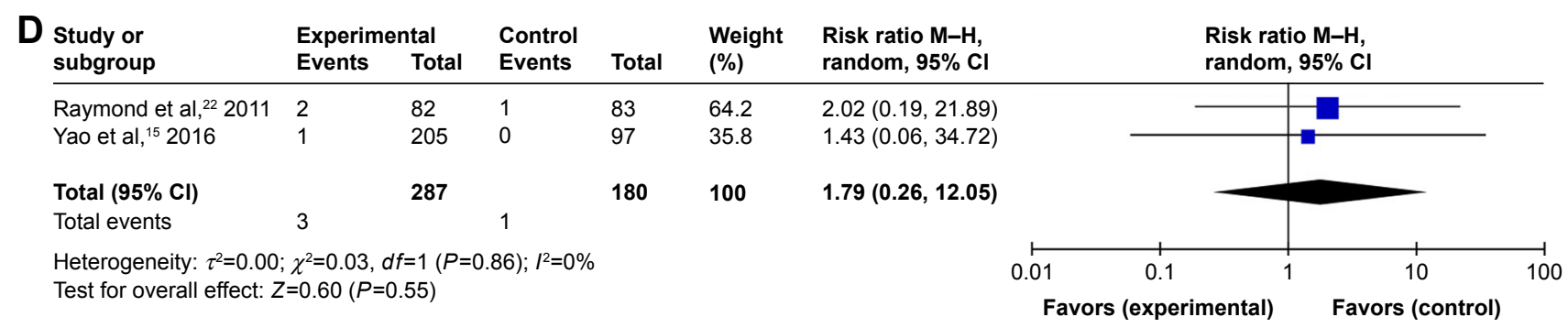

Figure 4 Relative risks of high-grade Gl events associated with nonoperative therapies.

Note: (A) Relative risk associated with nonoperative therapies of high-grade (A) diarrhea, (B) nausea, (C) vomiting, and (D) anorexia.

Abbreviation: $\mathrm{Gl}$, gastrointestinal.

in prognostic and therapeutic decisions for patients with pancreatic NET. ${ }^{27-29}$ To the best of our knowledge, this is the first meta-analysis focusing specifically on GI events associated with nonoperative therapies in NET patients, which widely include chemotherapy, molecular targeted therapy, IFN, SSAs, and PRRT. Except for patients with limited metastases who can undergo complete resection of the primary and metastatic tumors, nonoperative therapies 


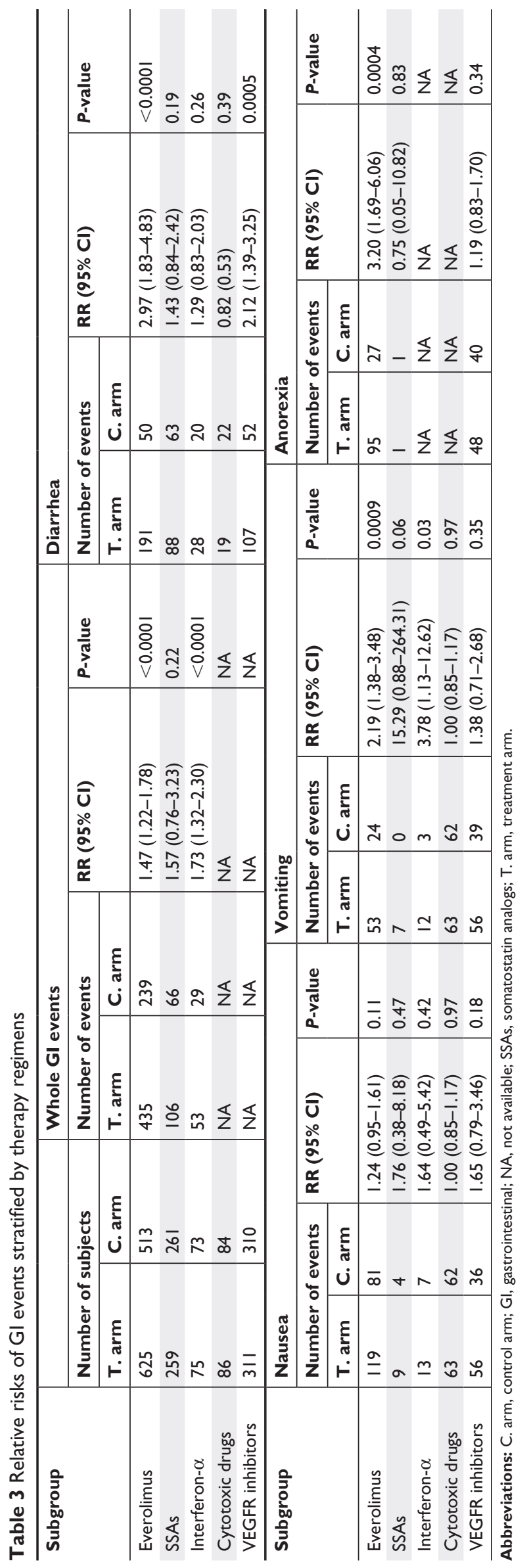

are the main recommendation for patients with locoregional advanced NETs or distant metastases. ${ }^{1}$

As most NETs upregulate the receptors of somatostatin (a peptide hormone), treatment with synthetic SSAs leads to obvious improvements in hormone-related symptoms and inhibits tumor growth. ${ }^{30,31}$ However, the expression of somatostatin receptors in the GI tract and pancreas can lead to significant disturbances in homeostasis induced by SSA treatment, as exemplified by the frequent occurrence of diarrhea in NET patients treated with the SSA lanreotide. ${ }^{21}$ In addition, targeted therapies (eg, everolimus and VEGFR inhibitors) lead to increased GI events, such as diarrhea, nausea, and vomiting. ${ }^{5,22}$ Cytotoxic chemotherapeutic agents (eg, doxorubicin and capecitabine), which are Category 3 recommendations for the treatment of NETs, can also markedly increase the frequency of diarrhea. ${ }^{32,33}$ Thus, GI events caused by these therapies can attenuate their pro-survival benefits and even lead to treatment discontinuation. ${ }^{5}$ Furthermore, diarrhea is one of the most common syndromes in NET patients with carcinoid syndrome. Before providing nonoperative therapies for NETs, pre-management of potential GI events with the conditions of nonoperative therapies should be ensured to promote patients' quality of life. However, the contribution of nonoperative therapies to the development of GI events is difficult to define when only individual RCTs are available, because they are not powered to clarify significant relationships.

This study pooled data from 17 RCTs to address the limited power of individual RCTs, and we found that nonoperative therapies generally increase the incidences and risks of GI events in NET patients. The RRs of diarrhea, nausea, vomiting, and anorexia associated with nonoperative therapies were 1.55 (95\% CI 1.21-1.98), 1.55 (95\% CI 1.14-2.10), 1.79 (95\% CI 1.14-2.10), and 1.87 (95\% CI 1.22-2.87), respectively. Among them, diarrhea was the most common GI event (33.1\%), and anorexia was the least common GI event $(16.6 \%)$ in the experimental groups. These results were largely consistent with the reported rates of GI events in several double-blind RCTs that tested sunitinib, everolimus, and SSAs in NET patients. ${ }^{5,21,22}$ Because high-grade adverse events often lead to therapy termination, we also evaluated the risks of high-grade GI events associated with nonoperative therapies. The data showed that the differences in the risks of high-grade diarrhea and anorexia between the experimental and control arms were not significant, whereas there were slight increases in the risks of high-grade nausea and vomiting.

When stratified by therapeutic regimen, it was more likely to be referred by physicians concerning the optimal 
Table 4 Relative risks of $\mathrm{Gl}$ events stratified by tumor sites

\begin{tabular}{|c|c|c|c|c|c|c|c|c|c|c|c|c|c|}
\hline \multirow[t]{3}{*}{ Subgroup } & \multicolumn{8}{|c|}{ Diarrhea } & \multicolumn{5}{|c|}{ Nausea } \\
\hline & \multicolumn{2}{|c|}{$\begin{array}{l}\text { Number of } \\
\text { subjects }\end{array}$} & \multicolumn{2}{|c|}{$\begin{array}{l}\text { Number of } \\
\text { events }\end{array}$} & \multirow{2}{*}{\multicolumn{2}{|c|}{ RR $(95 \% \mathrm{CI})$}} & \multirow{2}{*}{\multicolumn{2}{|c|}{$P$-value }} & \multicolumn{3}{|c|}{$\begin{array}{l}\text { Number of } \\
\text { events }\end{array}$} & \multirow[t]{2}{*}{$\operatorname{RR}(95 \% \mathrm{Cl})$} & \multirow[t]{2}{*}{$P$-value } \\
\hline & T. arm & C. arm & T. arm & C. arm & & & & & \multicolumn{2}{|c|}{ T. arm } & C. arm & & \\
\hline Pancreatic NET & 647 & 656 & 231 & 132 & \multicolumn{2}{|c|}{1.77 (I.13-2.79) } & \multicolumn{2}{|c|}{0.01} & \multicolumn{2}{|c|}{112} & 86 & $1.39(0.92-2.10)$ & 0.12 \\
\hline Gastrointestinal NET & 358 & 250 & 69 & 22 & \multicolumn{2}{|c|}{$1.66(1.05-2.63)$} & \multicolumn{2}{|c|}{0.03} & \multicolumn{2}{|l|}{37} & 12 & $1.57(0.84-2.94)$ & 0.15 \\
\hline $\begin{array}{l}\text { Gastroenteropancreatic } \\
\text { NET }\end{array}$ & 415 & 409 & 95 & 60 & \multicolumn{2}{|c|}{$1.56(0.89-2.75)$} & \multicolumn{2}{|c|}{0.12} & \multicolumn{2}{|l|}{92} & 69 & $1.28(0.88-1.87)$ & 0.20 \\
\hline & & \multicolumn{6}{|c|}{ Vomiting } & \multicolumn{6}{|c|}{ Anorexia } \\
\hline \multirow{2}{*}{\multicolumn{2}{|c|}{ Subgroup }} & \multicolumn{2}{|c|}{ Number of events } & \multirow{2}{*}{\multicolumn{2}{|c|}{ RR (95\% Cl) }} & \multirow{2}{*}{\multicolumn{2}{|c|}{$P$-value }} & \multicolumn{4}{|c|}{ Number of events } & \multirow[t]{2}{*}{ RR $(95 \%$ CI) } & \multirow[t]{2}{*}{$P$-value } \\
\hline & & T. arm & C. arm & & & & & T. a & rm & C. & arm & & \\
\hline \multicolumn{2}{|l|}{ Pancreatic NET } & 125 & 93 & \multicolumn{2}{|c|}{$1.37(0.88-2.13)$} & 0.17 & & 84 & & 54 & & $1.48(0.8 \mathrm{I}-2.73)$ & 0.21 \\
\hline Gastrointestinal NET & & 52 & 11 & $4.68(2.5$ & 8-8.49) & $<0.00$ & & 32 & & 6 & & $2.52(1.09-5.83)$ & 0.03 \\
\hline Gastroenteropancreatic & NET & 70 & 41 & $2.16(0.8$ & $4-5.60)$ & 0.11 & & 30 & & 14 & & $2.08(1.13-3.83)$ & 0.02 \\
\hline
\end{tabular}

Note: $P$-value $<0.05$ indicated statistical significance.

Abbreviations: C. arm, control arm; GI, gastrointestinal; NET, neuroendocrine tumor; T. arm, treatment arm.

therapy option for an individual NET patient on the basis of the precaution for GI events (especially high-grade events) during therapeutic process. Everolimus led to high risks of diarrhea, vomiting, and anorexia; IFN- $\alpha$ increased the risk of vomiting; VEGFR inhibitors resulted in an increased risk of diarrhea; combination chemotherapy showed no difference in GI events compared with single-drug chemotherapy. Intriguingly, SSAs led to no significant difference in the risk of diarrhea; however, a recent double-blind, placebocontrolled RCT of the SSA lanreotide suggested that the most common adverse event was diarrhea (26\% in the lanreotide group and $9 \%$ in the placebo group). ${ }^{21}$ This discrepancy could be explained by the high heterogeneity $\left(I^{2}=78 \%\right)$ in this subgroup meta-analysis.

Furthermore, we evaluated the risks of GI events stratified by tumor site. Patients with pancreatic NET exhibited a high risk of diarrhea, without evidence of increased risk of other GI events. In addition, GI NET patients had a higher risk of diarrhea. Further, therapies significantly increased the risks of vomiting and anorexia in GI NET patients. It is reasonable to speculate that targeting therapy toward tumors in GI sites could further disturb the functions of the GI tract, resulting in enhanced risks of therapy-related GI events.

PRRT with ${ }^{177} \mathrm{Lu}$-Dotatate was newly approved in January 2018 for the treatment of advanced and metastatic gastroenteropancreatic NETs. Studies showed that this therapy was helpful to improve symptoms and remarkably increase the progression-free survival (PFS) and overall survival (OS) in NET patients. ${ }^{8,34,35}{ }^{177}$ Lu-Dotatate showed very limited longterm toxicity, with no incidences of therapy-related renal and hepatic failure during follow-up. ${ }^{35}$ However, it led to significant GI events, including nausea (59\% of patients) and vomiting (52\% of patients). ${ }^{8}$ This finding was derived from a trial in which patients were randomly allocated to a ${ }^{177} \mathrm{Lu}-$ Dotatate plus octreotide group or an octreotide group. Future placebo-controlled RCTs are needed to research the risks of GI events posed by ${ }^{177} \mathrm{Lu}$-Dotatate in NET patients.

Despite the number of trials included in this meta-analysis, our study has several limitations. First, it was based on data from trial reports, not on individual patient data; therefore, we were unable to clarify the confounding variables, such as comorbidities, previous therapeutic interventions, and concomitant therapies. The inherent confounders may underestimate, overestimate, or bias the direction of the risk estimates. Second, the standards for attributing various GI events were not the same across the trials; GI events were the secondary endpoints (rather than the primary endpoints) of the included trials, resulting in a great bias with regard to the reported incidence rates. Third, all of the included trials required participants to have generally adequate organ functions, which suggests that the rates of real-world GI events would be higher. Finally, the number of enrolled patients was still inadequate for a meta-analysis of SSAs- and ${ }^{177} \mathrm{Lu}-$ Dotatate-induced GI events, especially due to lack of data from placebo-controlled RCTs.

In conclusion, our findings indicate that nonoperative therapies in NET patients lead to significantly increased risks of any- and high-grade nausea and vomiting. Strikingly, everolimus led to high risks of diarrhea, vomiting, and anorexia; VEGFR inhibitors led to an increased risk of diarrhea; and GI NETs led to more GI events than pancreatic NETs. Thus, rigorous management of these adverse events is warranted to minimize adverse impacts on treatment outcomes and quality of life. Once NET patients are assigned 
to systematic treatments, those who are prone to or suffering from severe GI events are eligible for treatments with lesser GI disturbance.

\section{Disclosure}

The authors report no conflicts of interest in this work.

\section{References}

1. Shah MH, Goldner WS, Halfdanarson TR, et al. NCCN guidelines insights: neuroendocrine and adrenal tumors, version 2.2018. J Natl Compr Canc Netw. 2018;16(6):693-702.

2. Dasari A, Shen C, Halperin D, et al. Trends in the incidence, prevalence, and survival outcomes in patients with neuroendocrine tumors in the United States. JAMA Oncol. 2017;3(10):1335.

3. Kulke MH, Shah MH, Benson AB, et al. Neuroendocrine tumors, version 1.2015. J Natl Compr Canc Netw. 2015;13(1):78-108.

4. Vinik A, Bottomley A, Korytowsky B, et al. Patient-reported outcomes and quality of life with sunitinib versus placebo for pancreatic neuroendocrine tumors: results from an international phase iii trial. Target Oncol. 2016;11(6):815-824.

5. Pavel ME, Hainsworth JD, Baudin E, et al. Everolimus plus octreotide long-acting repeatable for the treatment of advanced neuroendocrine tumours associated with carcinoid syndrome (RADIANT-2): a randomised, placebo-controlled, phase 3 study. Lancet. 2011; 378(9808):2005-2012.

6. Moertel CG, Hanley JA, Johnson LA. Streptozocin alone compared with streptozocin plus fluorouracil in the treatment of advanced isletcell carcinoma. N Engl J Med. 1980;303(21):1189-1194.

7. Arnold R, Rinke A, Klose KJ, et al. Octreotide versus octreotide plus interferon-alpha in endocrine gastroenteropancreatic tumors: a randomized trial. Clin Gastroenterol Hepatol. 2005;3(8):761-771.

8. Strosberg J, El-Haddad G, Wolin E, et al. Phase 3 Trial of ${ }^{177} \mathrm{Lu}$-Dotatate for Midgut Neuroendocrine Tumors. N Engl J Med. 2017;376(2): 125-135.

9. Moher D, Liberati A, Tetzlaff J, et al. Preferred reporting items for systematic reviews and meta-analyses: the PRISMA statement[J]. Annals of Internal Medicine. 2009;151(4):264-269.

10. Higgins J, Green S. Cochrane handbook for systematic reviews of interventions Version 5.1.0. The Cochrane Collaboration[J]. Confidence intervals, 2011.

11. Higgins JP, Thompson SG. Quantifying heterogeneity in a metaanalysis. Stat Med. 2002;21(11):1539-1558.

12. Higgins JP, Thompson SG, Deeks JJ, Altman DG. Measuring inconsistency in meta-analyses. BMJ. 2003;327(7414):557-560.

13. Begg CB, Mazumdar M. Operating characteristics of a rank correlation test for publication bias. Biometrics. 1994;50(4):1088-1101.

14. Egger M, Smith GD, Schneider M, Minder C. Bias in meta-analysis detected by a simple, graphical test. BMJ. 1998;316(7129):469-471.

15. Yao JC, Fazio N, Singh S, et al. Everolimus for the treatment of advanced, non-functional neuroendocrine tumours of the lung or gastrointestinal tract (RADIANT-4): a randomised, placebo-controlled, phase 3 study. Lancet. 2016;387(10022):968-977.

16. Yao JC, Pavel M, Lombard-Bohas C, et al. Everolimus for the treatment of advanced pancreatic neuroendocrine tumors: overall survival and circulating biomarkers from the randomized, phase III RADIANT-3 study. J Clin Oncol. 2016;34(32):3906-3913.

17. Fazio N, Buzzoni R, Baudin E, et al. A phase II study of BEZ235 in patients with everolimus-resistant, advanced pancreatic neuroendocrine tumours. Anticancer Res. 2016;36(2):713.

18. Rinke A, Müller HH, Schade-Brittinger C, et al. Placebo-controlled, double-blind, prospective, randomized study on the effect of octreotide LAR in the control of tumor growth in patients with metastatic neuroendocrine midgut tumors: a report from the PROMID Study Group. J Clin Oncol. 2009;27(28):4656-4663.
19. Wolin EM, Hu K, Hughes G, et al. Safety, tolerability, pharmacokinetics, and pharmacodynamics of a long-acting release (LAR) formulation of pasireotide (SOM230) in patients with gastroenteropancreatic neuroendocrine tumors: results from a randomized, multicenter, open-label, phase I study. Cancer Chemother Pharmacol. 2013;72(2):387-395.

20. Kulke MH, Ruszniewski P, Van Cutsem E, et al. A randomized, openlabel, phase 2 study of everolimus in combination with pasireotide LAR or everolimus alone in advanced, well-differentiated, progressive pancreatic neuroendocrine tumors: COOPERATE-2 trial. Ann Oncol. 2017; 28(6):1309-1315.

21. Caplin ME, Pavel M, Ćwikła JB, et al. Lanreotide in metastatic enteropancreatic neuroendocrine tumors. $N$ Engl J Med. 2014;371(3): 224-233.

22. Raymond E, Dahan L, Raoul JL, et al. Sunitinib malate for the treatment of pancreatic neuroendocrine tumors. N Engl J Med. 2011; 364(6):501-513.

23. Niccoli P, Raoul J, Bang Y, et al. Updated safety and efficacy results of the phase III trial of sunitinib (SU) versus placebo (PBO) for treatment of pancreatic neuroendocrine tumors (NET)[J]. Journal of Clinical Oncology. 2010;28(15_suppl):4000.

24. Phan AT. Randomized phase II study of everolimus (E) versus everolimus plus bevacizumab (E+B) in patients (Pts) with locally advanced or metastatic pancreatic neuroendocrine tumors. Clinical Advances in Hematology \& Oncology. 2015;13(6 Suppl 7):8-10.

25. Faiss S, Pape UF, Böhmig M, et al. Prospective, randomized, multicenter trial on the antiproliferative effect of lanreotide, interferon alfa, and their combination for therapy of metastatic neuroendocrine gastroenteropancreatic tumors - the International Lanreotide and Interferon Alfa Study Group[J]. Journal of Clinical Oncology. 2003;21(14):2689-2696.

26. Meyer T, Qian W, Caplin ME, et al. Capecitabine and streptozocin \pm cisplatin in advanced gastroenteropancreatic neuroendocrine tumours. Eur J Cancer. 2014;50(5):902-911.

27. Broder MS, Beenhouwer D, Strosberg JR, Neary MP, Cherepanov D. Gastrointestinal neuroendocrine tumors treated with high dose octreotideLAR: a systematic literature review. World J Gastroenterol. 2015; 21(6):1945-1955.

28. Partelli S, Cirocchi R, Crippa S, et al. Systematic review of active surveillance versus surgical management of asymptomatic small nonfunctioning pancreatic neuroendocrine neoplasms. Br J Surg. 2017; 104(1):34-41.

29. Pezzilli R, Partelli S, Cannizzaro R, et al. Ki-67 prognostic and therapeutic decision driven marker for pancreatic neuroendocrine neoplasms (PNENs): A systematic review. Adv Med Sci. 2016;61(1):147-153.

30. Oberg KE, Reubi JC, Kwekkeboom DJ, Krenning EP. Role of somatostatins in gastroenteropancreatic neuroendocrine tumor development and therapy. Gastroenterology. 2010;139(3):742-753.

31. Pyronnet S, Bousquet C, Najib S, Azar R, Laklai H, Susini C. Antitumor effects of somatostatin. Mol Cell Endocrinol. 2008;286(1-2): 230-237.

32. Chong CR, Wirth LJ, Nishino M, et al. Chemotherapy for locally advanced and metastatic pulmonary carcinoid tumors. Lung Cancer. 2014;86(2):241-246.

33. Mitry E, Walter T, Baudin E, et al. Bevacizumab plus capecitabine in patients with progressive advanced well-differentiated neuroendocrine tumors of the gastro-intestinal (GI-NETs) tract (BETTER trial) - a phase II non-randomised trial. Eur J Cancer. 2014;50(18):3107-3115.

34. Hörsch D, Ezziddin S, Haug A. Peptide receptor radionuclide therapy for neuroendocrine tumors in Germany: first results of a multi-institutional cancer registry. Recent Results Cancer Res. 2013;194(2):457-465.

35. Brabander T, van der Zwan WA, Teunissen JJM, et al. Long-Term Efficacy, Survival, and Safety of $\left[{ }^{177}\right.$ Lu-DOTA ${ }^{0}$, Tyr $\left.^{3}\right]$ octreotate in Patients with Gastroenteropancreatic and Bronchial Neuroendocrine Tumors. Clin Cancer Res. 2017;23(16):4617-4624. 


\section{Supplementary material}

A

Egger's test for diarrhea

- Metabias loger seloger, egger

Note: Data input format theta se_theta assumed.

Egger's test for small-study effects: Regress standard normal deviate of intervention
effect estimate against its standard error

Number of studies $=16$

\begin{tabular}{lllllll}
\multicolumn{2}{l}{ Number of studies $=16$} & \multicolumn{4}{r}{$r$} \\
\hline Std_Eff & Coef. & Std. Err. & $\boldsymbol{t}$ & $\boldsymbol{P}>|\boldsymbol{t}|$ & $\mathbf{( 9 5 \%} \mathrm{Cl})$ \\
\hline Slope & 0.2590915 & 0.2484044 & 1.04 & 0.315 & -0.2736829 & 0.7918658 \\
Bias & 0.6718425 & 0.9858039 & 0.68 & 0.507 & -1.442497 & 2.786182 \\
\hline \multicolumn{2}{l}{ Test of HO: no small-study effects } & \multicolumn{3}{c}{$P=0.507$}
\end{tabular}

C

Egger's test for vomiting

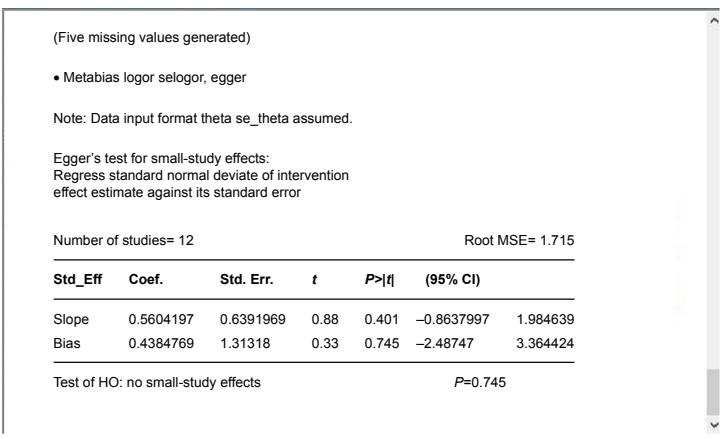

B

Egger's test for nausea

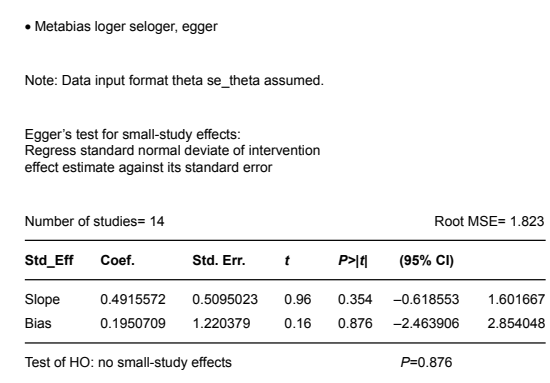

D

Egger's test for anorexia

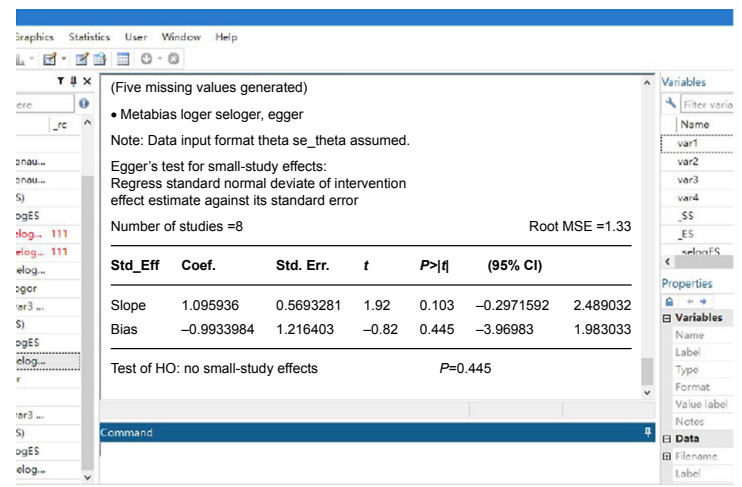

Figure SI Begg's test for the association between nonoperative therapies and risks of Gl events.

Abbreviation: Gl, gastrointestinal.

\section{Publish your work in this journal}

OncoTargets and Therapy is an international, peer-reviewed, open access journal focusing on the pathological basis of all cancers, potential targets for therapy and treatment protocols employed to improve the management of cancer patients. The journal also focuses on the impact of management programs and new therapeutic agents and protocols on patient perspectives such as quality of life, adherence and satisfaction. The manuscript management system is completely online and includes a very quick and fair peer-review system, which is all easy to use. Visit http://www.dovepress.com/testimonials.php to read real quotes from published authors. 